\title{
RETTORE DI TUTTI: PAOLO MANTEGAZZA E LE FACOLTÀ UMANISTICHE
}

\author{
ANTONIO PADOA-SCHIOPPA (*)
}

SunTO. - Breve resoconto della molteplice attività di Paolo Mantegazza quale Rettore della Università di Milano, la quale sotto la sua guida ha conosciuto uno sviluppo delle strutture scientifiche e didattiche senza precedenti.

$* * *$

ABSTRACT. - A short survey of the personality and of the multiple achievements of the President, of the University of Milan, whose work has been seminal in developing research, teaching and library structures of students and professors.

Questa giornata di studi che l'Istituto Lombardo ha dedicato all'opera di Paolo Mantegazza ad un anno dalla scomparsa sta rivelando non solo la poliedricità della sua figura di scienziato ma la straordinaria ampiezza dell'area dei suoi interventi nel quarto di secolo nel quale egli ricoperse le funzioni di preside della Facoltà di medicina e poi di rettore dell'Università di Milano. Confesso che anche chi, come me, ne ha pur seguito per anni l'attività non aveva avuto, sino ad oggi, la percezione chiara della sua vera statura di protagonista della ricerca e di grande e sapiente organizzatore di una struttura tanto complessa quale è quella dell'università italiana.

Era noto il fatto che mai, neppure nei lunghi anni di impegno rettorale, egli aveva tralasciato di tenersi al corrente del progresso degli studi nel suo settore di farmacologia; e che così continuò a fare anche negli ultimi anni, prodigo di osservazioni e di consigli. Ma sorprende quanto vari siano stati i campi nei quali ha spaziato la sua ricerca nel

(*) Istituto Lombardo Accademia di Scienze e Lettere, Università degli Studi di Milano, Italia. E-mail: antonio.padoaschioppa@gmail.com 
corso dei decenni e per oltre mezzo secolo: una caratteristica che diviene sempre più rara in un'età di così accentuata specializzazione in quasi tutti i campi del sapere non solo scientifico ma anche umanistico.

Ciò su cui vorrei brevemente intrattenervi qui riguarda invece la sua opera di rettore, che ho potuto seguire lungo l'intero arco del suo rettorato, durante il quale io sono stato ininterrottamente preside della Facoltà di Giurisprudenza. Sono stati anni e decenni - specie dalla metà degli anni Ottanta alla metà degli anni Novanta - nei quali la popolazione universitaria non solo cresceva, ma proprio nel settore giuridico segnava tassi di aumento eccezionali, solo gradualmente discesi negli anni più recenti. Si è giunti allora ad un afflusso vicino e persino superiore alle 5000 matricole annue, cui faceva fronte un corpo docente di circa 40 professori, con poco più di 50 ricercatori, necessariamente e fortunatamente coadiuvati da un piccolo esercito di assistenti volontari, spesso avvocati o magistrati o notai, operanti agli esami (centinaia per appello) a titolo volontario e gratuito. Nonostante la dispersione degli iscritti nei primi due anni, i laureati in legge eccedevano annualmente le 1000 unità. Non è difficile immaginare il peso didattico connesso con le tesi di laurea, che allora impegnavano lo studente per circa sei mesi, concludendosi quasi sempre al quinto o al sesto anno di corso e non di rado anche più tardi, specie per gli studenti lavoratori.

In questi anni, sotto la guida del rettore Mantegazza, la Facoltà di Giurisprudenza ha ottenuto un innesto di risorse (oltre 20 nuove cattedre) senza precedenti; ha moltiplicato le aule, per un tempo usufruendo anche della platea del Teatro Carcano, attrezzata per le serate teatrali ma accogliente, al mattino, oltre mille studenti; ha dato spazio in via $\mathrm{S}$. Antonio, dove oltre alle aule ed a prestigiose strutture per gli uffici si è aperta la bellissima Sala napoleonica, fiore all'occhiello dell'Ateneo per conferenze ed anche, a lungo, per le lauree; ha infine acquisito e ristrutturato, sempre per impulso del rettore, lo stabile di via Mercalli per aule, seminari, scuole di specializzazione e uffici, gestito in comune con le Facoltà di Lettere e di Scienze politiche. Ma soprattutto, la Facoltà giuridica ha triplicato i corsi di laurea istituendo in un primo tempo i poli di Bicocca e di Como entro un'unica Facoltà, poi trasformandoli in nuove Facoltà nei due nuovi Atenei di Milano Bicocca e di Insubria. E' stato un progetto ambizioso e di successo che chi vi parla ha seguito da vicino per Giurisprudenza. Di Milano Bicocca ci ha informato efficacemente or ora chi è stato protagonista dello sviluppo di questo nuovo Ateneo ormai affermato a livello nazionale, Marcello Fontanesi. 
La Facoltà di Lettere ha in questi anni conseguito risultati non meno significativi. Il polo di Sant'Alessandro ha valorizzato efficacemente un edificio storico, debitamente ristrutturato, dove hanno ora sede i Dipartimenti di Lingue e letterature straniere. Lo scavo dei cortili interni ha consentito la creazione di spazi adeguati per Biblioteche di alta qualità, quali quelle del Dipartimento di Scienze storiche e di Filosofia, così come pure - per Giurisprudenza - la Sala di Common Law, unica nel suo genere in Italia.

A sua volta, la Facoltà di Scienze politiche è stata completamente modificata nelle strutture didattiche e logistiche con il completo rinnovo della sede in via Conservatorio. Una scelta intelligente di apertura agli studenti lavoratori, anche in ore serali, una moderna Biblioteca centrale e un insieme di risorse adeguate, con particolare cura per il comparto di Sociologia, poi in larga misura confluito nella sede di Bicocca, ne hanno profondamente rinnovato l'offerta didattica e le strutture scientifiche.

Il vertice di questa politica di rinnovo e di crescita è costituito così almeno io ritengo e non sono certo il solo - dalla ristrutturazione degli spazi del Sotto-crociera e della Crociera sforzesca di via Festa del Perdono. Un deposito in disuso ed uno spazio prima adibito a eventi occasionali è stato trasformato in due grandi Sale di consultazione con migliaia di volumi a vista, destinate agli studenti e ai ricercatori delle due Facoltà umanistiche site nella sede centrale, Lettere e Giurisprudenza, ma non solo ad esse. Centinaia di studenti, da ormai quasi vent'anni, lavorano quotidianamente in questi spazi, che un tempo furono sede delle corsie dell'Ospedale maggiore. Mai il preside Enrico Decleva e il sottoscritto, che per molti anni si erano battuti per ottenere questo risultato, vi sarebbero giunti senza il sostegno convinto del rettore Mantegazza, che non esitò ad affrontare anche le vivaci resistenze della sua stessa Facoltà, favorevole a mantenere la destinazione precedente. Non a caso ho intitolato questo breve ricordo di lui designandolo come il "rettore di tutti".

Oggi, quando un docente straniero o una delegazione universitaria vengono a visitare la sede di Festa del Perdono, immancabilmente il vertice dell'ammirazione viene raggiunto allorché li si conduce a vedere la Crociera gremita di studenti al lavoro nella sede che per secoli era stata una struttura di sofferenza, la grande corsia dei malati, allora all'avanguardia per modernità di impostazione.

E non basta. E' stato sotto la guida di Paolo Mantegazza che si è 
proceduto anche allo scavo del grande cortile del Settecento, dove hanno trovato spazio decine di migliaia di volumi e di riviste giuridiche a scaffale aperto e dove giornalmente lavorano ricercatori e centinaia di studenti. I posti di studio - sia attrezzati che liberi - per gli studenti delle Facoltà umanistiche sono aumentati, durante gli anni del rettorato di Mantegazza, di oltre mille unità: una crescita senza precedenti.

Può sembrare, quella degli spazi, una politica universitaria di semplice buona amministrazione. Ma a torto. La qualità del lavoro didattico e scientifico - dipende in misura importante dalla razionalità degli spazi nei quali esso si svolge (persino la qualità del lavoro degli organi collegiali dipende dall'ambiente e addirittura dalla forma e dalle dimensioni del tavolo per le riunioni). Questo insegna l'esperienza.

Un aspetto rilevante della personalità di Mantegazza rettore, giustamente sottolineato da Enrico Decleva, sta nel fatto che egli, a differenza di altri, non mirava tanto a realizzare progetti da lui stesso direttamente concepiti, ma piuttosto a realizzare quelle iniziative che, a lui proposte da altri, gli sembrassero degne di attuazione. In questo è stato davvero grande. Né gli innovativi cinque poli di Medicina, né i nuovi Atenei, né le tante realizzazioni di cui oggi si è detto sarebbero state possibili senza questo atteggiamento di ascolto - molto raro in chi esercita un potere - coniugato con una infinita pazienza nelle riunioni collegiali (anche di questo ho avuto innumeri esperienze nel corso degli anni). Tra i tanti esempi possibili, mi piace ricordare il sostegno da lui dato all'Istituto Lombardo, che oggi ci ospita, in anni nei quali i contributi pubblici per le Accademie si erano (e si sono..) purtroppo ridotti al lumicino: la convenzione del Lombardo con la Statale si è estesa ad altri Atenei lombardi ed ha consentito alla nostra Accademia di continuare la sua attività bisecolare a pieno regime.

Della capacità di ascolto di Mantegazza ho già detto, ma va aggiunto che il suo impegno diretto e personale nei casi in cui qualcuno gli chiedeva aiuto per ragioni di malattia sua o di un parente era senza limiti. Se una delle segretarie gli riferiva in tempo reale (come lui voleva) la telefonata di un collega, anche da lui non conosciuto, che richiedeva un consiglio medico per sé o per altri, il rettore interrompeva per qualche minuto qualunque riunione per rispondere immediatamente, attivandosi per aiutarlo: non ricordo forse nessun Senato accademico in cui questo non sia avvenuto almeno una volta.

In questo, come in altri comparti del suo operare, svolgeva un ruolo centrale la fede religiosa. Ed emergeva inoltre, nel suo rapporto 
con le sofferenze altrui, l'esperienza personale del dolore, che nella vita non gli è stato risparmiato. Il suo cristianesimo coniugava un atteggiamento di realismo disincantato per le debolezze umane con la convinzione di fondo che alla fine le forze del male "non praevalebunt".

Tali erano le capacità operative e le doti eminenti, i carismi, della persona che in tanti abbiamo avuto il privilegio di conoscere e che oggi ricordiamo con rimpianto e gratitudine. 